\title{
Measuring Global Financial Linkages: A Network Entropy Approach
}

\author{
Daeyup Lee ${ }^{1}$ and Hail Park ${ }^{2, *}$ \\ 1 Economic Research Institute, Bank of Korea, Seoul 04531, Korea \\ 2 Department of International Business and Trade, Kyung Hee University, Seoul 02447, Korea \\ * Correspondence: hailpark@khu.ac.kr; Tel.: +82-2-961-2170
}

Received: 23 July 2019; Accepted: 23 August 2019; Published: 28 August 2019

\begin{abstract}
This paper measures the diversity of highly-connected financial networks using network entropy, and policy-related findings emerge from this research. With respect to the time variation of network entropy, international diversification of the global financial network constructed from foreign claims of international banks has decreased since the financial crisis of 2007-2008, while foreign claims among 20 reporting countries have concentrated more on core countries, such as the US and UK, since 2009. This change is more vividly captured by network entropy due to an unprecedented drop in the measurement. The results suggest that network entropy has promising potential in the financial market.
\end{abstract}

Keywords: network entropy; global financial networks

JEL Classification: F34

\section{Introduction}

The recent financial crises highlight the importance of interconnected global financial markets in the global economy [1]. With the literature on financial networks growing rapidly, the empirical works can be divided into two strands. One of those strands focuses on data-based investigations of financial networks to identify and understand their features. For example, [2] used the BIS (Bank for International Settlements) consolidated banking sector statistics to investigate cross-border exposure and provided several vulnerability measures. Among them, the borrower concentration ratio, which is based on the Herfindahl index, and is similar to entropy in the sense that two measures gauge the extent of the diversification of lending. A higher borrower concentration ratio may be interpreted as a higher contagion risk. [3] examined the BIS locational statistics by focusing on flows rather than on exposures. By employing network metrics, they found that connectivity tends to decline during and after financial crises. [4] built a bank-level global financial network and identified a systematic effect of recessions and banking crises on the global banking network. [5] extended a cross-border network of the banking sectors in the Euro area to include sector networks of each country and highlight the tradeoff between efficiency and stability in financial networks.

The research of the second strand centers around a slightly different question. How do the financial networks transmit exogenous shocks to financial markets? To respond to this question, researchers have widely used network simulation techniques. For instance, [6] studied cross-border contagion risk during the period 1999-2006 by using the cross-border exposure data of 17 countries from the BIS banking statistics. They adopted the approach developed in [7] and found that the contagion risk increased during the period. [8] derived a tipping point condition of contagion dynamics in financial networks. Their simulation experiments revealed that the concentration and complexity of financial networks are important amplifiers of financial fragility. 
Moreover, global financial networks are naturally related to another important topic, that of global liquidity. For example, [9] emphasized the role of global banks in the transmission of global liquidity across borders. In this context, it is important to develop quantitative methods for analyzing financial networks. The current literature on financial networks focuses primarily on the connectivity of networks via the number of links. The network structures of international financial markets are rapidly becoming more complex due to the advancement of international financial integration. Thus, it is apparent that finer tools are needed to analyze these highly connected networks.

In this regard, this paper aims to contribute to understanding the network structure of global financial markets by providing a network entropy measure of network diversity, or international diversification, proposed by [10] and readily applicable to highly connected networks. Also, there may be regional heterogeneity in network diversification. Countries in Asia, Africa, and the Middle East might have both, a lower volatility of banking flows, and a more diverse set of creditors. Given that the main source of variation comes from the financial crisis that emerged in Europe, and is mainly exposed to west European parent banks, most of which were badly affected during the crisis.

Roughly speaking, network entropy is composed of two elements, namely, eigenvector centrality and (Shannon) entropy. Eigenvector centrality, which, in general, depends on network structures, is one of the measures of node centrality in network analysis. Several papers advocate the usefulness of eigenvector centrality in the analysis of financial networks ([11-14]). In turn, entropy has been used as a measure of diversification in the economic literature. For example, [15] have developed an entropy measure of corporate diversification. In the context of the analysis of financial networks, entropy can be viewed as a measure of the diversification of the links of a node in a network. By combining eigenvector centrality and entropy, the entropy of a node can be determined, and the entropies of nodes can reveal the heterogeneity of nodes even if they are completely connected. Finally, network entropy is a weighted sum of the entropies of the nodes in a network. Thus, network entropy is a network-wide measure of diversification that takes into account network structures by incorporating eigenvector centrality.

Several papers, similar to this paper, use eigenvector centrality to analyze networks. In contrast, [11] use the dominant left eigenvector of a (modified) weighted adjacency matrix to rank participants in the Canadian large-value transfer system with respect to their daily liquidity holdings. Similarly, [12] employ the dominant left eigenvector of a matrix of IPO (Initial Public Offering) flows to assess the importance of international financial centers in attracting global IPOs. [13,14], however, study financial derivatives from the perspective of network analysis and propose a super-spreader tax based on the dominant right eigenvector. In comparison to these papers, our paper goes further by applying an entropy measure based on eigenvector centrality.

In this paper, we apply the measure of network entropy to the BIS global financial network database to study highly connected global financial networks. Based on the new measure of network diversity, the research question in this paper is as follows. How did the network structure of global banking networks among core countries (i.e., reporting countries) evolve during the global financial crisis of 2007-2009 with respect to diversification? Policy-related findings emerge from this research. Regarding the time variation of network entropy, the international diversification of the global financial network constructed from foreign claims of international banks retreats after the financial crisis of 2007-2008. While foreign claims among 20 reporting countries have become more concentrated on core countries such as the US and the UK from 2009, the change is more vividly captured by network entropy, which exhibits an unprecedented drop in the measure. This finding demonstrates that network entropy is a more sensitive measure of the diversity of a financial network due to the inclusion of information about financial network structures that is captured by eigenvector centrality.

The paper is organized as follows. Section 2 presents the data and the methodology used in this paper. Section 3 reports the main results of the paper, and Section 4 provides the conclusion. 


\section{Data and Methodology}

\subsection{Data}

The BIS consolidated banking statistics were used in this study. This paper focused on the foreign claims, which are defined as the sum of cross-border claims plus foreign offices' local claims in all currencies [16]. The sample period of foreign claims was from the first quarter of 2006 to the third quarter of 2012. Of the reporting countries, 20 countries were chosen because of data availability (According to the BIS consolidated banking statistics, there was only one break reported during the sample period (2006-2012). The United States increased its reporting population in the first quarter of 2009). Figure 1 depicts the trends of the foreign claims of the 20 countries. Figure 1 reveals that the subnetwork of the 20 reporting countries has stagnated more severely than has the whole network. However, Figure 1 does not provide information about whether there are changes in the structures of global financial linkages. Therefore, to answer the question, it may be beneficial to examine the network properties of the linkages.

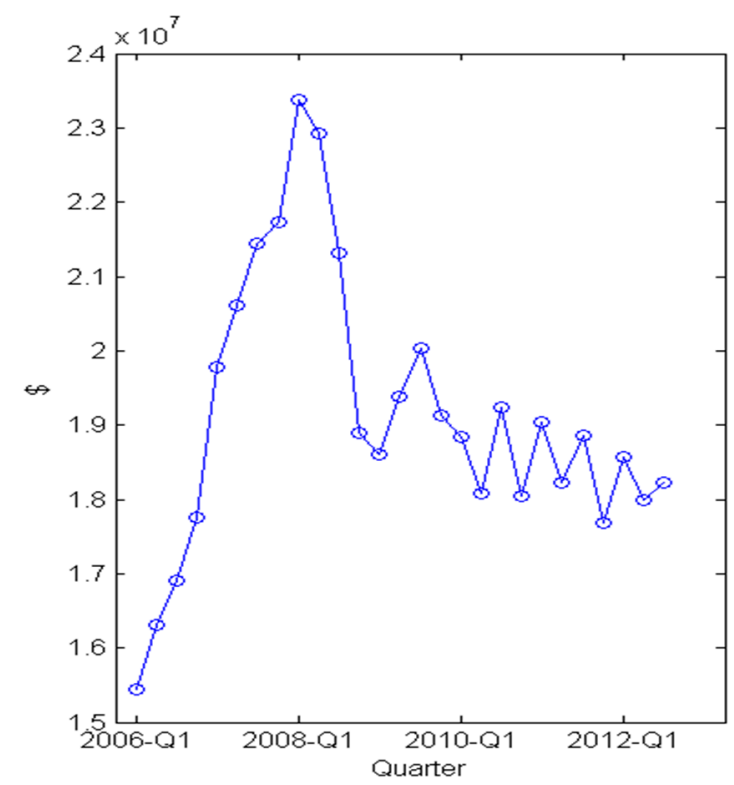

Figure 1. Total foreign claims (2006 Q1-2012 Q3): The 20 countries include Austria, Australia, Belgium, Canada, Chile, France, Germany, Greece, India, Ireland, Italy, Japan, Netherlands, Portugal, Spain, Sweden, Switzerland, Turkey, United Kingdom, and United States.

Figure 2 presents global financial networks constructed based on the foreign claims of the 20 reporting countries (For visualization of financial networks, we used the software, financial network analytics (FNA), developed by Kimmo Soramäki which is available at www.fna.fi). The size of the nodes is proportional to the amount of lending by a country, and the thickness of the link lines is determined by the fraction of the foreign claims of one country on another country compared to its total foreign claims. The figures indicate that there have been changes in the relative shares of the volumes of lending by the 20 countries. For instance, the nodes of the US and Japan are larger in 2012 than they are in 2006. To see the changes more precisely, quantitative measures of the networks, the concept of entropy, and related tools are required. 


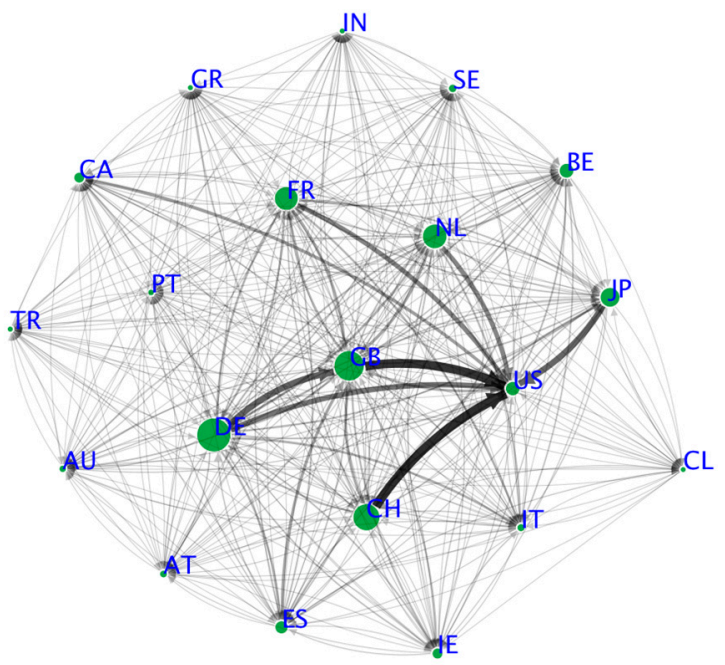

(a) 2006 Q1

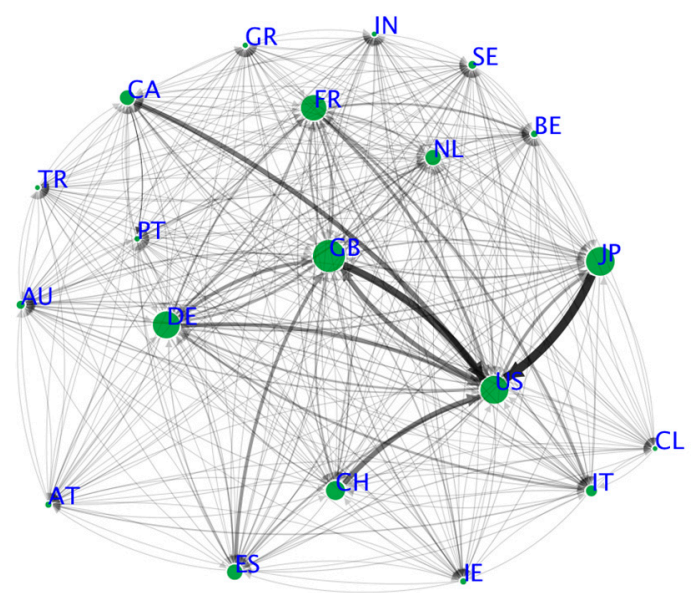

(b) 2012 Q3

Figure 2. Global financial networks: AT: Austria, AU: Australia, BE: Belgium, CA: Canada, $\mathrm{CH}$ : Switzerland, CL: Chile, DE: Germany, ES: Spain, FR: France, GB: United Kingdom, GR: Greece, IE: Ireland, IN: India, IT: Italy, JP: Japan, NL: Netherlands, PT: Portugal, SE: Sweden, TR: Turkey, and US: United States.

\subsection{Methodology}

A network $(\mathrm{N}, \mathrm{A})$ is defined by a set of nodes $\mathrm{N}=\{1,2, \ldots, \mathrm{n}\}$ and an adjacency matrix $\mathrm{A}=\left(\alpha_{i j}\right)_{1 \leq i, j \leq n}$, where the subscripts, $i$ and $j$, indicate nodes of the network. A node, or vertex, is the basic unit of the network. Nodes can be individuals, groups, cities, web pages, etc. In this paper, a node represents a country in the global financial market. Nodes are connected by edges, i.e., links. An edge score $\alpha_{i j}$ of the adjacency matrix A corresponds to the strength of the interaction between node $i$ and node $j$. In general, the edge score $\alpha_{i j}$ can take either a binary value, as in an unweighted network, or a real value, as in a weighted network. If $\alpha_{i j} \neq \alpha_{i j}$ for some pair $(i, j)$, then the corresponding network is called a directed network. Otherwise, it is called an undirected network. In this paper, $\alpha_{i j}$ and $\alpha_{j i}$ represent foreign claims of country i on country $\mathrm{j}$ and vice versa. It is clear that in general, $\alpha_{i j} \neq \alpha_{j i}$. Therefore, the network is a weighted and directed network. Moreover, since the foreign claims exhibit non-negative values, the adjacency matrix $\mathrm{A}$ is a non-negative matrix. If a financial network is a complete network, then the adjacency matrix A is a primitive matrix. 


\subsubsection{Transformation of Adjacency Matrices into Stochastic Matrices}

Adjacency matrices must be transformed into stochastic matrices to apply the concept of (Shannon) entropy. In this paper, we adopt the method proposed by [10]. However, before introducing the method, a more straightforward normalization, i.e., a fraction of total foreign claims, must be considered for comparison.

Method 1. Given an adjacency matrix A, a stochastic matrix $\hat{P}=\left(\hat{p}_{i j}\right)$ is defined as follows:

$$
\hat{p}_{i j}=\frac{\alpha_{i j}}{\sum_{j} \alpha_{i j}}
$$

Method 1 treats each node uniformly. In other words, Method 1 does not take into account the possible heterogeneity of nodes. Accordingly, Method 1 will serve as a benchmark.

Method 2. In the literature on networks, several measures of centrality have been developed. Among them is the eigenvector centrality proposed by [17], which is based on neighbors' characteristics. The basic idea of eigenvector centrality is that a node's centrality depends on the centralities of its neighbors. In particular, [17] assumes that a node's centrality is proportional to the weighted sum of the edge scores where weights are provided by centrality scores. Given adjacency matrix A, the assumption yields a system of homogenous linear equations for the unknown variables, i.e., the centrality scores, such that $v=\left\{v_{1}, v_{2}, \ldots, v_{\mathrm{n}}\right\}$, as in [17]:

$$
\sum_{j} \alpha_{i j} v_{j}=\lambda v_{i}, 1 \leq i, j \leq n
$$

where $\lambda$ is a proportionality constant. The centrality scores should be non-negative. Equation (2) illustrates why the proposed centrality is called the eigenvector centrality. The pair $(\lambda, v)$ corresponds to an eigenvalue-eigenvector pair of the adjacency matrix A.

Suppose that an adjacency matrix $\mathrm{A}$ is a non-negative and primitive matrix. Then, the Perron-Frobenius theorem ([18], Theorem 1.1) indicates that there exists a dominant eigenvalueeigenvector pair, $\left(\lambda_{\max }, v_{\max }\right)$ such that

$$
\mathrm{A} v_{\max }=\lambda_{\max } v_{\max }
$$

where both $\lambda_{\max }$ and $v_{\max }$ are positive. $v_{\max }$ is uniquely determined up to scale. Then, $v_{\max }$ is normalized, yielding $\sum_{j} v_{\max }(j)=1 . v_{\max }(j)$ is described as a measure of relative contribution made by node $j$ to the network. In global financial works, the right eigenvector $v_{\max }(j)$ represents the relative contribution made by country $\mathrm{j}$ to lending money. Then, given an adjacency matrix A, Method 2 defines a stochastic matrix $\mathrm{P}^{*}=\left(p_{i j}^{*}\right)$ as follows:

$$
p_{i j}^{*}=\frac{\alpha_{i j} v_{\max }(j)}{\lambda_{\max } v_{\max }(i)}, 1 \leq i, j \leq n
$$

\subsubsection{Network Entropy}

Several entropy measures have been used as a complexity measure of a graph ([19]). Following [10], we apply the well-known Shannon entropy formula. Given a discrete probability distribution $D=\left\{p_{1}\right.$, $\left.p_{2}, \ldots, p_{n}\right\}$, the formula is defined as follows:

$$
H(D)=-\sum_{i=1}^{n} p_{i} \log p_{i}
$$


Note that $H(D)$ can be interpreted as the expected value of the random variable $\log \left(\frac{1}{p}\right)$. In particular, from the perspective of financial network analysis, entropy can be described as a measure of diversification given that the formula allows a more evenly distributed random variable to obtain higher entropy.

Given a stochastic matrix $\left(P=\left(p_{i j}\right)\right)$ derived from an adjacency matrix of a network, the entropy $\left(H_{i}\right)$ of node $i$ is obtained by applying the formula to transition the probability distribution, which corresponds to the $i$ th row of the stochastic matrix $(\mathrm{P})$.

$$
H_{i}(P)=-\sum_{i=1}^{n} p_{i j} \log p_{i j}, 1 \leq i, j \leq n
$$

$H_{i}(P)$ measures the diversity of choices of node $i$. The network entropy $\left(H_{\text {network }}\right)$ is then defined as the weighted sum of the entropies of the nodes.

$$
H_{\text {network }}(P)=\sum_{i=1}^{n} \pi_{i} H_{i}
$$

where the weighting vector $\pi=\left\{\pi_{1}, \pi_{2}, \ldots, \pi_{n}\right\}$ is the unique invariant distribution of the corresponding stochastic matrix $(\mathrm{P})$.

$$
\pi \mathrm{P}=\pi
$$

If a stochastic matrix is ergodic, i.e., primitive, then the unique invariant distribution is well-defined ([20], Theorem 5.9). The left eigenvector $\pi$ is a measure of relative contributions made by countries borrowing money from global financial markets. In general, $H_{\text {network }}(P)$ quantifies the average diversity of the choices of the nodes. Particularly, in this paper, $H_{\text {network }}(P)$ is a measure of the international diversification in the global financial networks.

\section{Network Entropy}

Figure 3 depicts the networks associated with the stochastic matrices, which are constructed from the foreign claims of 20 countries. Accordingly, the figures provide snapshots of the global financial networks in 2006 Q1 and 2012 Q3 from the perspective of a network entropy approach. The width of each edge in the networks is proportional to the components of the stochastic matrices, and the size of each node is determined by the invariant distributions of the stochastic matrices. Compared with Figure 2, Figure 3 reveals the crucial roles played by leading countries in incorporating the heterogeneity of nodes in terms of eigenvector centrality. Other countries lend disproportionately large amounts of money to leading countries. Also, Figure 3 uncovers a notable shrinkage in Germany's weight and a sizable increase in the weight of the US in the global financial networks.

Figure 4 presents the time variations of the network entropies of foreign claims. The left panel of Figure 4 represents the network entropies computed by Method 1, and the right panel depicts the network entropies calculated by Method 2. Note that the network entropy obtained from Method 2 dropped sharply from the first quarter of 2009, while the network entropy from Method 1 experienced only moderate changes compared to Method 2. Figure 4 illustrates the point that Method 2 may better detect changes in network structures since it incorporates changes in the centrality of nodes, whereas Method 1 does not.

To see which countries are most responsible for these changes in network entropy in Method 2, the components of network entropy for the G5 countries should be examined because they are the major players in the global markets. Figure 5 exhibits the trends of the eigenvector centralities, entropies, and invariant probabilities of the G5 countries. The upper panel unveils the sizable rise of the eigenvector centrality of the US from 2009 Q1. It also displays the steady increase of the eigenvector centrality of Japan. In contrast, the eigenvector centralities of France and Germany have decreased particularly since the 2010 European economic crisis. In particular, although Germany has the highest eigenvector 
centrality in 2006 Q1, Japan, the UK, and the US have higher eigenvector centralities in 2012 Q3 than Germany. It seems that these changes in eigenvector centrality are essential in understanding the different results from the two methods because the eigenvector centrality is the only distinctive feature of Method 2. The middle panel displays the entropies of five countries. The most severe declines appear in the cases of Japan and the UK. It is noted that these two countries are more strongly connected with the US than are France and Germany. The observation, combined with the rising eigenvector centrality of the US, helps to understand the drivers of the remarkable drops of the entropies of Japan and the UK. In terms of invariant probabilities, the bottom panel reveals a rising tendency for the US and Japan and a descending tendency for France and Germany, which implies that more money is flowing into the US and Japan.

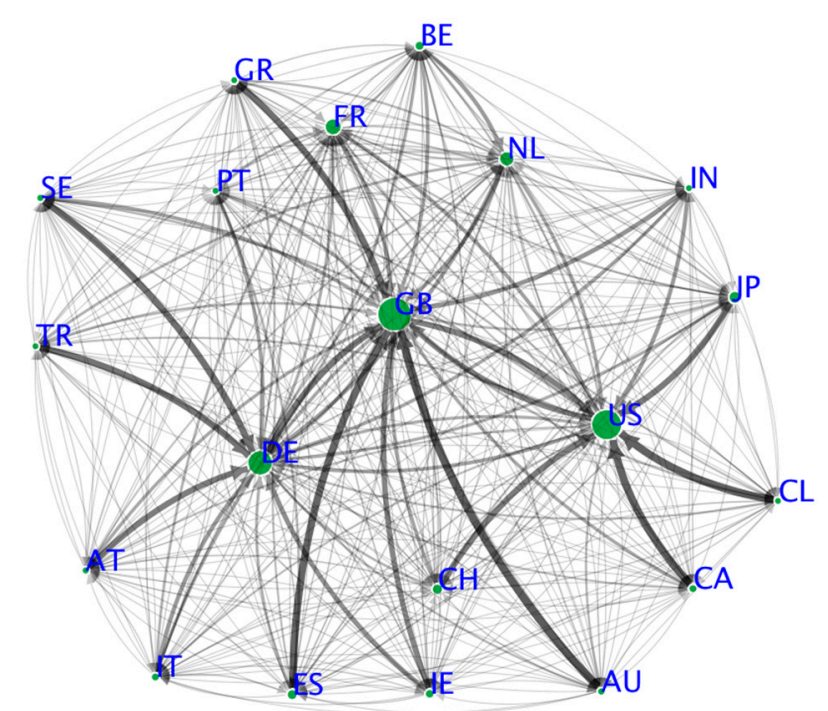

(a) 2006 Q1

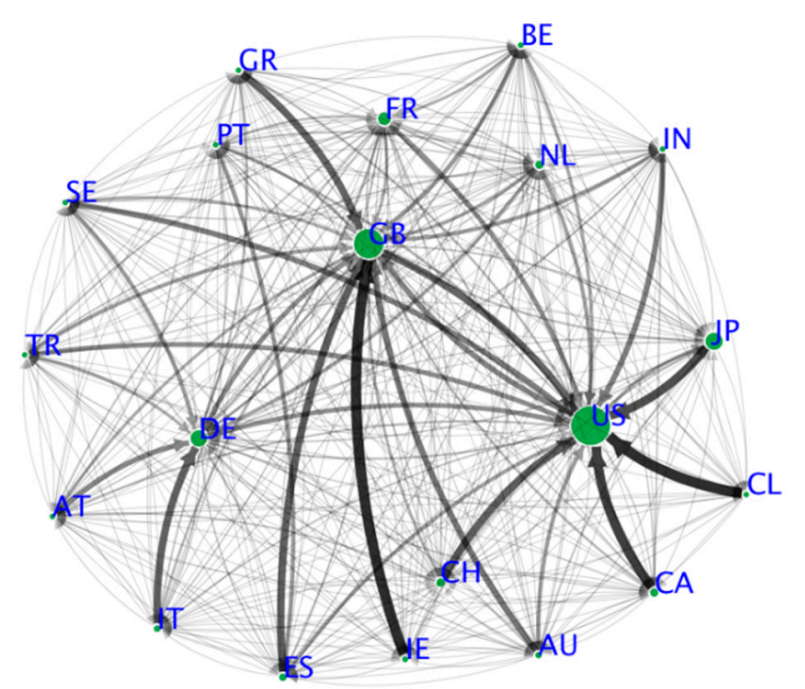

(b) $2012 \mathrm{Q} 3$

Figure 3. Global financial networks using network entropy: The width of each edge in the network is proportional to the components of the stochastic matrices, and the size of each node is determined by the invariant distributions for the stochastic matrices. 


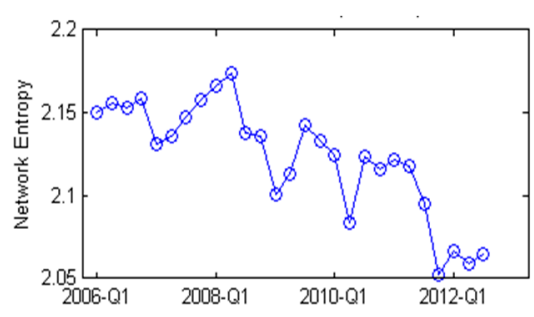

(Method 1)

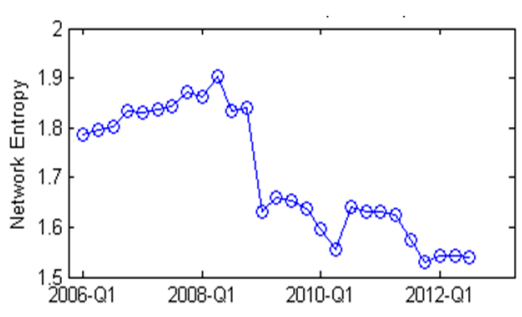

(Method 2)

Figure 4. Network entropies of foreign claims.
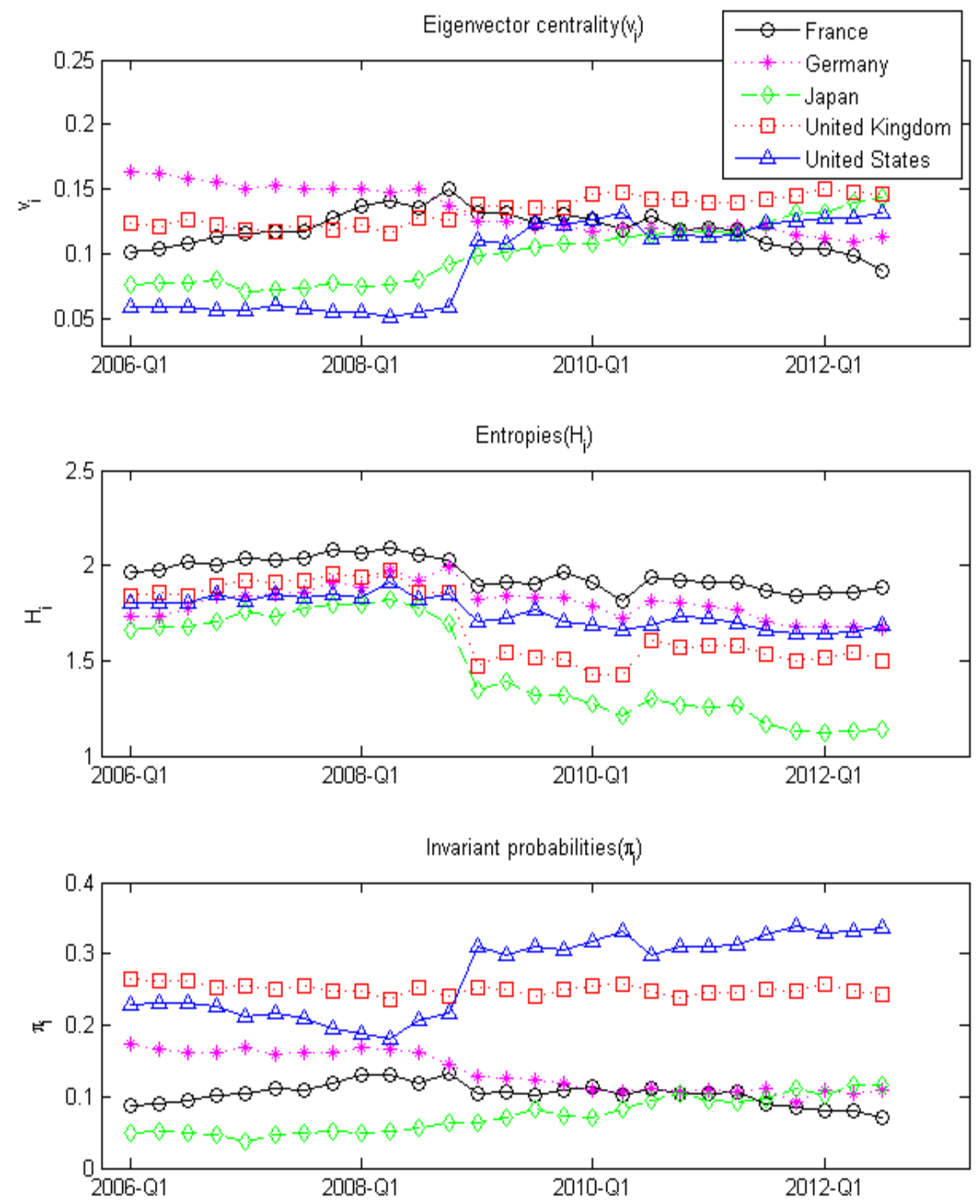

Figure 5. Components of network entropy for the G5 countries.

Table 1 provides a more comprehensive perspective of the components of network entropy by providing snapshots for four periods. Table 1 includes the periods 2008 Q2 and 2009 Q1 for comparison in addition to the starting and ending periods of the sample. From Table 1, it is evidenced that in addition to France and Germany, the eigenvector centralities of Belgium and Netherlands also declined sizably during the sample period. Regarding the entropies, it is interesting to note that only the entropies of Greece and Italy decreased between 2006 Q1 and 2008 Q2. Finally, the invariant probabilities of Belgium, Austria, and Italy, in addition to France and Germany, diminished during the sample period reflecting the European debt crisis, which implies that the importance of these countries 
in international borrowing decreased. Overall, the analysis of the components of network entropy provides a detailed picture of how the network structures of the global financial markets evolved during the financial crises.

Table 1. Components of network entropy ${ }^{1}$.

\begin{tabular}{|c|c|c|c|c|c|c|c|c|c|c|c|c|}
\hline & \multicolumn{4}{|c|}{ Eigenvector Centrality $\left(v_{i}\right)$} & \multicolumn{4}{|c|}{ Entropy of a Node $\left(H_{i}\right)$} & \multicolumn{4}{|c|}{ Invariant Probabilities $\left(\pi_{i}\right)$} \\
\hline & 2006-Q1 & 2008-Q2 & 2009-Q1 & 2012-Q3 & 2006-Q1 & 2008-Q2 & 2009-Q1 & 2012-Q3 & 2006-Q1 & 2008-Q2 & 2009-Q1 & 2012-Q3 \\
\hline AT & 0.01 & 0.01 & 0.01 & 0.01 & 1.74 & 1.82 & 1.83 & 1.79 & 0.00 & 0.00 & 0.00 & 0.00 \\
\hline $\mathrm{AU}$ & 0.01 & 0.01 & 0.01 & 0.02 & 1.31 & 1.33 & 1.27 & 1.43 & 0.00 & 0.01 & 0.00 & 0.01 \\
\hline $\mathrm{BE}$ & 0.06 & 0.07 & 0.04 & 0.01 & 1.87 & 1.89 & 1.89 & 1.87 & 0.02 & 0.03 & 0.01 & 0.00 \\
\hline $\mathrm{CA}$ & 0.03 & 0.02 & 0.04 & 0.07 & 1.32 & 1.35 & 0.90 & 0.80 & 0.01 & 0.01 & 0.01 & 0.03 \\
\hline $\mathrm{CH}$ & 0.12 & 0.09 & 0.09 & 0.09 & 1.59 & 1.78 & 1.38 & 1.30 & 0.03 & 0.02 & 0.02 & 0.03 \\
\hline CL & 0.00 & 0.00 & 0.00 & 0.00 & 1.35 & 1.65 & 0.78 & 0.58 & 0.00 & 0.00 & 0.00 & 0.00 \\
\hline $\mathrm{DE}$ & 0.16 & 0.15 & 0.13 & 0.11 & 1.73 & 1.97 & 1.83 & 1.67 & 0.17 & 0.17 & 0.13 & 0.11 \\
\hline ES & 0.05 & 0.05 & 0.05 & 0.06 & 1.41 & 1.58 & 1.29 & 1.12 & 0.03 & 0.03 & 0.03 & 0.02 \\
\hline FR & 0.10 & 0.14 & 0.13 & 0.09 & 1.97 & 2.09 & 1.90 & 1.88 & 0.09 & 0.13 & 0.10 & 0.07 \\
\hline GB & 0.12 & 0.12 & 0.14 & 0.15 & 1.84 & 1.97 & 1.47 & 1.50 & 0.26 & 0.24 & 0.25 & 0.24 \\
\hline GR & 0.00 & 0.00 & 0.00 & 0.00 & 1.70 & 1.41 & 1.54 & 1.42 & 0.00 & 0.00 & 0.00 & 0.00 \\
\hline IE & 0.04 & 0.04 & 0.03 & 0.01 & 1.60 & 1.66 & 1.56 & 0.59 & 0.02 & 0.02 & 0.02 & 0.00 \\
\hline IN & 0.00 & 0.00 & 0.00 & 0.00 & 1.79 & 1.97 & 1.63 & 1.58 & 0.00 & 0.00 & 0.00 & 0.00 \\
\hline IT & 0.02 & 0.05 & 0.04 & 0.03 & 1.98 & 1.25 & 1.47 & 1.37 & 0.01 & 0.04 & 0.02 & 0.01 \\
\hline JP & 0.08 & 0.08 & 0.10 & 0.14 & 1.66 & 1.82 & 1.34 & 1.14 & 0.05 & 0.05 & 0.06 & 0.12 \\
\hline NL & 0.11 & 0.09 & 0.07 & 0.05 & 1.89 & 2.02 & 1.94 & 1.88 & 0.07 & 0.06 & 0.03 & 0.03 \\
\hline PT & 0.00 & 0.01 & 0.00 & 0.00 & 2.08 & 2.01 & 2.06 & 1.93 & 0.00 & 0.00 & 0.00 & 0.00 \\
\hline SE & 0.02 & 0.02 & 0.01 & 0.02 & 1.45 & 1.57 & 1.61 & 1.36 & 0.00 & 0.00 & 0.00 & 0.00 \\
\hline $\mathrm{TR}$ & 0.00 & 0.00 & 0.00 & 0.00 & 1.62 & 1.79 & 1.67 & 1.56 & 0.00 & 0.00 & 0.00 & 0.00 \\
\hline US & 0.06 & 0.05 & 0.11 & 0.13 & 1.80 & 1.91 & 1.70 & 1.68 & 0.23 & 0.18 & 0.31 & 0.34 \\
\hline
\end{tabular}

${ }^{1}$ AT: Austria, AU: Australia, BE: Belgium, CA: Canada, CH: Switzerland, CL: Chile, DE: Germany, ES: Spain, FR: France, GB: United Kingdom, GR: Greece, IE: Ireland, IN: India, IT: Italy, JP: Japan, NL: Netherlands, PT: Portugal, SE: Sweden, TR: Turkey, US: United States.

\section{Concluding Remarks}

This paper proposes network entropy as a tool for measuring the diversity of highly connected financial networks. The computation of network entropy hinges on eigenvector centrality and Shannon entropy. We find some supporting evidence for the advantages of this approach in that the measures that incorporate eigenvector centrality provide additional information about changes in foreign claims over other simple measures that do not include eigenvector centrality. Meanwhile, the global financial crisis of 2007-2008 highlighted the importance of indirect financial linkages across countries. To capture indirect aspects of financial connections, quantitative measures need to be sensitive to network characteristics of counterparty countries. The results in this study show that network entropy is tailor-made for the purpose.

Policy-related findings emerge from this research. With respect to the time variation of network entropy, international diversification of the global financial network constructed from foreign claims of international banks declines after the financial crisis of 2007-2008. Furthermore, it is found that foreign claims among 20 reporting countries have concentrated more on core countries such as the US and the UK since 2009. In other words, more money has been flowing into big players in global markets, searching for safe heavens. This change is more vividly captured by network entropy, which reveals an unprecedented decline. This finding illustrates that network entropy is a more sensitive measure for the diversity of a financial network due to the inclusion of information about financial network structures captured by eigenvector centrality. For policy-makers, properly monitoring the evolving features of financial networks is essential for ensuring financial stability. Thus, the results suggest that network entropy has promising potential in the financial market.

Author Contributions: All authors contributed equally to this work and all authors have read and approved the final manuscript. The views expressed herein are those of the authors and do not necessarily reflect the official views of Bank of Korea. When reporting or citing this paper, the authors' names should be explicitly stated. 
Funding: This research received no external funding.

Conflicts of Interest: The authors declare no conflict of interest.

\section{References}

1. Yellen, J.L. Interconnectedness and Systemic Risk: Lessons from the Financial Crisis and Policy Implications; Speech delivered at the American Economic Association/American Finance Association Joint Luncheon: San Diego, CA, USA, 2013.

2. Weistroffer, C.; Mo“bert, J. Monitoring Cross-Border Exposure: A Primer on how to Exploit the BIS Banking Statistics; Deutsche Bank Research Current Issues; Deutsche Bank Research: Frankfurt am Main, Germany, 2010.

3. Minoiu, C.; Reyes, J.A. A Network Analysis of Global Banking: 1978-2010. J. Financ. Stab. 2013, 9, 168-184. [CrossRef]

4. Hale, G. Bank Relationships, Business Cycles, and Financial Crises. J. Int. Econ. 2012, 88, 312-325. [CrossRef]

5. Castrén, O.; Rancan, M. Macro-Networks: An Application to the Euro Area Financial Accounts; ECB Working Paper No. 1510; European Central Bank: Frankfurt am Main, Germany, 2013.

6. Degryse, H.; Elahi, M.A.; Penas, M.F. Cross-Border Exposures and Financial Contagion. Int. Rev. Financ. 2010, 10, 209-240. [CrossRef]

7. Upper, C.; Worms, A. Estimating Bilateral Exposures in the German Interbank Market: Is There a Danger of Contagion? Eur. Econ. Rev. 2004, 48, 827-849. [CrossRef]

8. Gai, P.; Haldane, A.; Kapadia, S. Complexity, Concentration and Contagion. J. Monet. Econ. 2011, 58, 453-470. [CrossRef]

9. Shin, H.S. Global Banking Glut and Loan Risk Premium. IMF Econ. Rev. 2012, 60, 155-192. [CrossRef]

10. Demetrius, L.; Manke, T. Robustness and Network Evolution-An Entropic Principle. Physica A 2005, 346, 682-696. [CrossRef]

11. Bech, M.L.; Chapman, J.T.; Garratt, R.J. Which Bank Is the “Central” Bank? J. Monet. Econ. 2010, 57, $352-363$. [CrossRef]

12. Cetorelli, N.; Peristiani, S. Prestigious Stock Exchanges: A Network Analysis of International Financial Centers. J. Bank. Financ. 2013, 37, 1543-1551. [CrossRef]

13. Markose, S.; Giansante, S.; Shaghaghi, A.R. 'Too Interconnected to Fail' Financial Network of US CDS Market: Topological Fragility and Systemic Risk. J. Econ. Behav. Organ. 2012, 83, 627-646. [CrossRef]

14. Markose, S.M. Systemic Risk from Global Financial Derivatives: A Network Analysis of Contagion and Its Mitigation with Super-Spreader Tax; IMF Working Paper WP/12/282; International Monetary Fund: Washington, DC, USA, 2012.

15. Jacquemin, A.P.; Berry, C.H. Entropy Measure of Diversification and Corporate Growth. J. Ind. Econ. 1979, 27, 359-369. [CrossRef]

16. BIS. Guidelines to the International Consolidated Banking Statistics; Bank for International Settlements: Basel, Switzerland, 2012.

17. Bonacich, P. Factoring and Weighting Approaches to Status Scores and Clique Identification. J. Math. Sociol. 1972, 2, 113-120. [CrossRef]

18. Seneta, E. Non-Negative Matrices and Markov Chains; Springer: Berlin/Heidelberg, Germany, 2006.

19. Dehmer, M.; Mowshowitz, A. A History of Graph Entropy Measures. Inf. Sci. 2011, 181, 57-78. [CrossRef]

20. Koralov, L.; Sinai, Y.G. Theory of Probability and Random Processes; Springer: Berlin/Heidelberg, Germany, 2012. 